\title{
Contrast enhanced ultrasound of a rare case of Peliosis hepatis
}

\author{
Alexander Loizides ${ }^{1}$, Bernhard Glodny' ${ }^{1}$, Heinz Zoller², Bettina Gudrun Zelger ${ }^{3}$, Daniel Junker ${ }^{1}$, \\ Benjamin Henninger ${ }^{1}$, Daniel Putzer ${ }^{1}$, Hannes Gruber ${ }^{1}$
}

${ }^{1}$ Department of Radiology, ${ }^{2}$ Department of Internal Medicine II, Gastroenterology \& Hepatology, ${ }^{3}$ Institute of Pathology, Medical University Innsbruck, Innsbruck, Austria

\begin{abstract}
Peliosis hepatis is a rare benign disorder of the liver, histologically characterized by blood-filled cystic cavities of various sizes and irregular shapes, communicating with the hepatic sinusoids. Only a few cases of peliosis hepatis have been described using contrast enhanced ultrasound showing admittedly various dynamic enhancement patterns. We present a case of peliosis hepatis with a typical target-sign enhancement depicted by means of contrast enhanced ultrasound.
\end{abstract}

Keywords: peliosis hepatis; ultrasound; contrast agent

\section{Introduction}

Peliosis hepatis is a rare benign vascular disease of the liver characterized by dilatation of hepatic sinusoids and blood-filled parenchymal cysts. The term "peliosis" arises from the Greek word "pelios" i.e. livid-purple color referring to the color of the liver parenchyma. Most cases of peliosis hepatis are incidental findings in asymptomatic patients. The etiology and pathogenesis of peliosis hepatis remain unclear: early descriptions reported a relation to tuberculosis or leprosy, nowadays the disease is described in relation to drugs (anabolic steroids, contraceptives or other hormonal treatment) [1] or even in the context of human immunodeficiency virus-related bacterial infections [2]. Also cases have been described associated with agents administered after kidney transplantation. Histologically the internal surface of the blood-filled cystic spaces may or not be covered with endothelium.

Received 18.04.2016 Accepted 15.05.2016

Med Ultrason

2017, Vol. 19, No 1, 114-116

Corresponding author: Alexander Loizides, $\mathrm{MD}, \mathrm{PhD}$ Medical University Innsbruck, Department of Radiology

Anichstrasse 35, 6020 Innsbruck, Austria

Phone: $+43 / 512 / 504 / 22761$

Fax: $+43 / 512 / 504 / 22758$

E-mail: alexander.loizides@i-med.ac.at
In radiological studies various dynamic enhancement patterns have been described, making the diagnose challenging $[3,4]$ : due to this variable imaging findings peliosis hepatis can mimic other hepatic conditions including malignant lesions such as hepatocellular carcinoma or liver metastases which influence the further treatment of the patient if misdiagnosed. The most common enhancement pattern described is a globular central enhancement in the arterial phase with a centrifugal progression and homogeneous enhancement on delayed phase.

We present a rare case of peliosis hepatis which was suspected on initial MRI and consecutive contrast enhanced ultrasound (CEUS) and confirmed by means of ultrasound guided biopsy.

\section{Case report}

A 66 year old asymptomatic woman was referred to our department for an MRI examination for suspect liver lesions which were seen in a routine sonographic examination of the abdomen. There was no history of early infections and no immunosuppressive or other drugs. The father died from colorectal cancer.

MRI of the liver was performed on a 1.5 Tesla MRI Scanner (Avanto Siemens). The standard sequence protocol included T2 turbo spin echo (TSE), T1 TSE, chemical shift imaging, diffusion weighted imaging (DWI) $(b=50$, 400,800 ), dynamic contrast enhanced images (VIBE in 
arterial, porto-venous and delayed phase). Hepatobiliary specific contrast media was used for imaging (Gd-EOBDTPA). Hepatobiliary phase images were obtained one hour after contrast media administration. MRI images showed multiple ill-defined nodules within the right hepatic lobe, hyperintense in T2 TSE, hypointense in T1 TSE. and without diffusion restriction. After contrast media administration, the nodules showed centrifugal contrast enhancement with equilibration after 5 minutes. On hepatobiliary phase images they were hypointense. Chemical shift images revealed no content of fat. The surrounding liver parenchyma showed very inhomogeneous contrast enhancement with multiple areas of adjacent hypo- and hyperperfusion, which was suspicious for the presence of a vascular disease (fig 1). Although this observation combined with the unusual presentation of the multiple nodules (centrifugal enhancement, missing diffusion restriction and hypointensity on hepatobiliary specific phase images) was suggestive for peliosis hepatis, a malignancy could not be excluded.

An additional CEUS with subsequent ultrasound guided biopsy was therefore planed in the interdisciplinary tumor-conference board.

The ultrasound examination was performed on a Philips iU22® (Philips, Bothell, Washington, USA) using a 5-1 MHz curved array transducer. On the initial greyscale ultrasound a $2.5 \times 1.7 \mathrm{~cm}$ hypoechogenic lesion was evident in the hepatic segment VIII. CEUS was performed using a contrast harmonic imaging technique with a second-generation blood pool contrast agent (SonoVue ${ }^{\circledR}$, Bracco, Milan, Italy). The standard MI setting of 0.06 was applied as provided by the vendor. After a single bolus in-

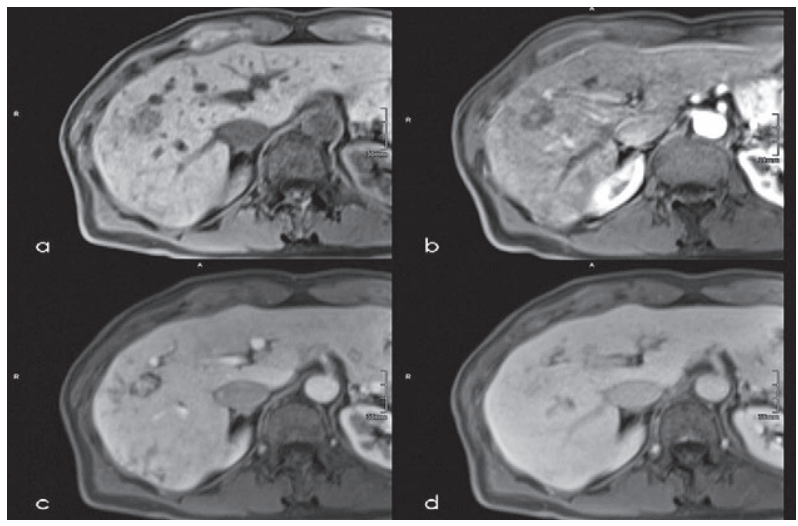

Fig 1. MRI in dynamic phases before (a) and after intravenous administration of Gadolinium (b-d). The lesion presents spotty hypointense on native T1-weighted images consistent with sinusoidal dilatation (a). Arterial phase shows a central enhancement (b) followed by a centrifugal spread in the portal venous phase (c). Delayed imaging (d) reveals incompete equilibration with suggested "branching" appearance caused by the vascular component. jection of $4.8 \mathrm{ml}$ of the contrast agent $5 \mathrm{ml}$ of normal saline solution were injected via a 20 -gauge intravenous cannula placed in the antecubital vein. The enhancement pattern of the lesions was documented on a continuous 3-min movie loop. In the early arterial phase a central enhancement was observed in the lesion with a progredient centrifugal spread and a homogeneous enhancement on delayed phase (fig 2). All obtained images were stored in our institutional Agfa ${ }^{\circ}-P A C S$ (Agfa AG, Mortsel, Belgium).

The subsequent ultrasound guided biopsy was performed by a specialist in interventional ultrasound with more than 8 years experience following a standardized procedure using a spring loaded $16 \mathrm{G} / 16 \mathrm{~cm}$ tru-cut ${ }^{\mathrm{TM}}$ biopsy needle in coaxial technique. The biopsy was performed under continuous real-time ultrasound guidance in a free-hand "in-plane-needle approach" to guarantee

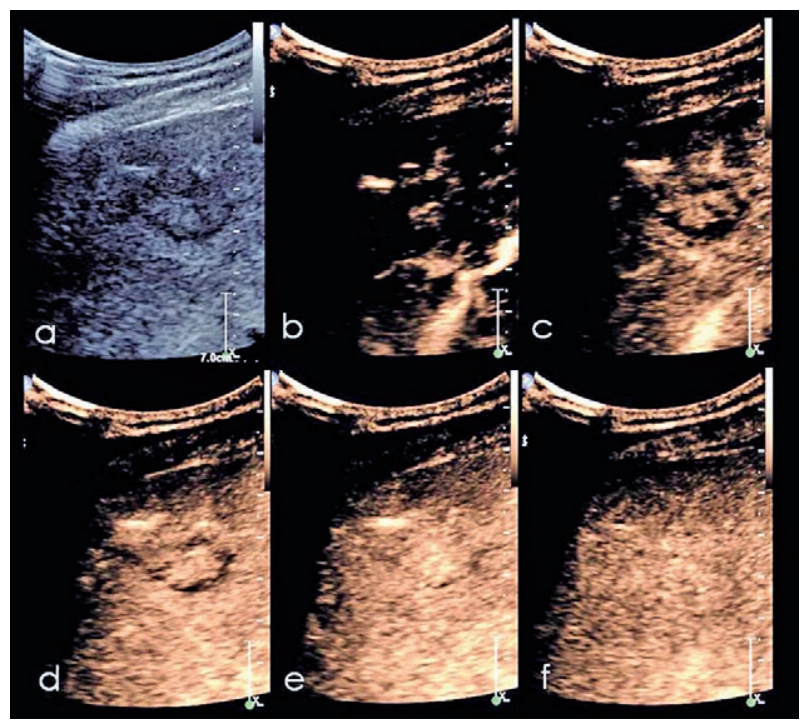

Fig 2. Grey scale ultrasound depicting a hypoechoic lesion (a) and contrast enhanced ultrasound after intravenous administration of Sonovue ${ }^{\circledR}$ (b-f) showing a central enhancement in the early arterial phase with a centrifugal spread and a homogeneous enhancement on delayed phase.

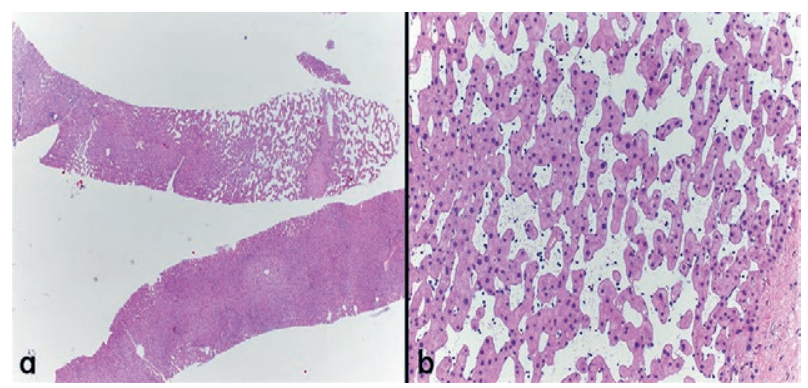

Fig 3. Liver core biopsies showing adjacent to normal liver parenchyma sinusoidal dilatation forming irregular spaces with thinning of hepatic cell cords without endothelial lining and fibrosis - magnification x 4 (a) and x 20 (b). 
for a clear biopsy of the respectively intended area. Three cores were obtained for the histopathological work-up. The histopathologic examination revealed the typical features of parenchymal peliosis hepatis: irregular bloodfilled cavities with communication through the sinusoids (fig 3).

\section{Discussions}

Peliosis hepatis is a rare benign disease entity with unknown incidence and prevalence. The pathogenesis of peliosis remains unclear: an obstruction of hepatic outflow at the level of the sinusoids is suggested, which leads to a breakdown of sinusoidal borders with consecutive dilatation of the hepatic lobules central vein and cavity formation. Many cases are incidental findings which are detected radiologically, in surgical procedures, or autopsy. The radiological evaluation of peliosis hepatis is challenging, as different enhancement patterns have been described. The most frequently described pattern is the so called target-sign, an early arterial contrast accumulation in the center of the lesion progressing in a centrifugal manner $[3,4]$. In the delayed phases a homogeneous accumulation is observed without signs of a contrast washout. Cases have been though described with a centripetal contrast enhancement mimicking hepatic hemangiomas $[5,6]$ or cases with atypical enhancement patterns which were not suggestible for any typical hepatic lesion [7].

Although biopsy of a suspected peliosis hepatis should if possible be avoided because of a significant risk of bleeding [8], in the majority of the cases, as in our case, a biopsy was performed due to the rarity of the disease and the variety of its imaging appearance.

The use of CEUS has become an additional adjunct in the evaluation of focal liver lesions in the last decade: the most frequent liver tumor entities can be characterized with high diagnostic accuracy as has been evidenced in large multicenter studies $[9,10]$. Nevertheless the diagnosis of rare focal lesions often remains problematic as characteristic contrast enhancement and vascularity patterns are always addressed to frequent tumor entities.

In our knowledge only a few cases of peliosis hepatis have been described using CEUS $[5,11,12]$. In contrary to our presented centrifugal contrast spread, a peripheral ring enhancement in the arterial phase with centripetal filling and homogeneous hyperenhancement in the late phase was described in the case of Gronlykke et al [5] whereas a homogeneous hyperenhancement $(n=1)$ and isoenhancement $(\mathrm{n}=1)$ in the arterial phase with washout $(\mathrm{n}=2)$ during the portal or late phase were noted in the cases of Xu HX et al [11]. Schuldes et al described a peripheral hyperenhancement of the lesions with central but not peripheral wash-out in the late phase [12]. All of the above mentioned cases described various rather "atypical" enhancing patterns.

In our case a typical target-sign enhancement e.g. an early arterial contrast accumulation in the center of the lesion with a centrifugal filling and a homogeneous enhancement on late phase was depicted by means of CEUS.

\section{References}

1. Nadell J, Kosek J. Peliosis hepatis. Twelve cases associated with oral androgen therapy. Arch Pathol Lab Med 1977; 101:405-410.

2. Garcia-Tsao G, Panzini L, Yoselevitz M, West AB. Bacillary peliosis hepatis as a cause of acute anemia in a patient with the acquired immunodeficiency syndrome. Gastroenterology 1992;102:1065-1070.

3. Gouya H, Vignaux O, Legmann P, de Pigneux G, Bonnin A. Peliosis hepatis: triphasic helical CT and dynamic MRI findings. Abdom Imaging 2001;26:507-509.

4. Iannaccone R, Federle MP, Brancatelli G, et al. Peliosis hepatis: spectrum of imaging findings. AJR Am J Roentgenol 2006; 187:W43-W52.

5. Gronlykke L, Tarp B, Dutoit SH, Wilkens R. Peliosis hepatis: a complicating finding in a case of biliary colic. BMJ Case Rep 2013;2013. doi: 10.1136/bcr-2013-200539.

6. Steinke K, Terraciano L, Wiesner W. Unusual cross-sectional imaging findings in hepatic peliosis. Eur Radiol 2003;13:1916-1919.

7. Kim SH, Lee JM, Kim WH, Han JK, Lee JY, Choi BI. Focal peliosis hepatis as a mimicker of hepatic tumors: radiological-pathological correlation. J Comput Assist Tomogr 2007;31:79-85.

8. Tsirigotis P, Sella T, Shapira MY, et al. Peliosis hepatis following treatment with androgen-steroids in patients with bone marrow failure syndromes. Haematologica 2007;92:e106-e110.

9. Strobel D, Seitz K, Blank W, et al. Contrast-enhanced ultrasound for the characterization of focal liver lesions--diagnostic accuracy in clinical practice (DEGUM multicenter trial). Ultraschall Med 2008;29:499-505.

10. Tranquart F, Correas JM, Ladam Marcus V, et al. Real-time contrast-enhanced ultrasound in the evaluation of focal liver lesions: diagnostic efficacy and economical issues from a French multicentric study. J Radiol 2009;90:109-122.

11. Xu HX, Xie XY, Lu MD, et al. Unusual benign focal liver lesions: findings on real-time contrast-enhanced sonography. J Ultrasound Med 2008;27:243-254.

12. Schuldes M, Weickert U. Contrast-enhanced ultrasound in suspected liver metastases. Dtsch Med Wochenschr 2011;136:1255-1256. 